\title{
Periderm prevents pathological epithelial adhesions during embryogenesis
}

\author{
Rebecca J. Richardson, ${ }^{1,2}$ Nigel L. Hammond ${ }^{1,2}$ Pierre A. Coulombe, ${ }^{3}$ Carola Saloranta, ${ }^{4}$ Heidi O. Nousiainen, ${ }^{5}$ Riitta Salonen, ${ }^{6}$ \\ Andrew Berry, ${ }^{2}$ Neil Hanley, ${ }^{2}$ Denis Headon, ${ }^{7}$ Riitta Karikoski, ${ }^{8}$ and Michael J. Dixon ${ }^{1,2}$ \\ ${ }^{1}$ Faculty of Life Sciences and ${ }^{2}$ Faculty of Medical and Human Sciences, Manchester Academic Health Sciences Centre, University of Manchester, Manchester, United Kingdom. ${ }^{3}$ Department of Biochemistry \\ and Molecular Biology, Bloomberg School of Public Health, Johns Hopkins University, Baltimore, Maryland, USA. ํDepartment of Genetics, Helsinki University Central Hospital, Helsinki, Finland. \\ ${ }^{5}$ Public Health Genomics Unit, National Institute for Health and Welfare, Helsinki University Hospital, Helsinki, Finland. ${ }^{6}$ Department of Medical Genetics, Väestöliitto, Helsinki, Finland. ${ }^{7}$ The Roslin Institute \\ and Royal (Dick) School of Veterinary Studies, University of Edinburgh, Edinburgh, United Kingdom. ${ }^{8}$ Department of Pathology, Helsinki University Hospital, Helsinki, Finland.
}

\begin{abstract}
Appropriate development of stratified, squamous, keratinizing epithelia, such as the epidermis and oral epithelia, generates an outer protective permeability barrier that prevents water loss, entry of toxins, and microbial invasion. During embryogenesis, the immature ectoderm initially consists of a single layer of undifferentiated, cuboidal epithelial cells that stratifies to produce an outer layer of flattened periderm cells of unknown function. Here, we determined that periderm cells form in a distinct pattern early in embryogenesis, exhibit highly polarized expression of adhesion complexes, and are shed from the outer surface of the embryo late in development. Mice carrying loss-of-function mutations in the genes encoding IFN regulatory factor 6 (IRF6), IкB kinase- $\alpha$ (IKK $\alpha$ ), and stratifin (SFN) exhibit abnormal epidermal development, and we determined that mutant animals exhibit dysfunctional periderm formation, resulting in abnormal intracellular adhesions. Furthermore, tissue from a fetus with cocoon syndrome, a lethal disorder that results from a nonsense mutation in IKKA, revealed an absence of periderm. Together, these data indicate that periderm plays a transient but fundamental role during embryogenesis by acting as a protective barrier that prevents pathological adhesion between immature, adhesion-competent epithelia. Furthermore, this study suggests that failure of periderm formation underlies a series of devastating birth defects, including popliteal pterygium syndrome, cocoon syndrome, and Bartsocas-Papas syndrome.
\end{abstract}

\section{Introduction}

The mature epidermis consists of 4 distinct cellular layers that undergo a constant process of displacement and renewal as cells from the outermost, cornified layer are sloughed off $(1,2)$. This process relies on a stable population of cells in the innermost basal layer, which proliferate constantly to produce daughter cells committed to terminal differentiation $(3,4)$. Appropriate development of stratified, squamous, keratinizing epithelia, such as the epidermis and oral epithelia, therefore results in the generation of an outer barrier that prevents water loss, entry of toxins, and microbial invasion (4).

During development, the immature ectoderm initially consists of a single layer of mitotically active, undifferentiated, cuboidal epithelial cells that progress through a series of defined stratification and differentiation events to produce the mature epidermis. The first stratification event produces a secondary layer of cells known as the periderm (4). The periderm is a single layer of flattened cells that covers the developing epithelia with a continuous layer (4-6). The periderm is believed to persist on the surface of the developing epidermis throughout embryogenesis until shortly

Authorship note: Rebecca J. Richardson and Nigel L. Hammond contributed equally to this work.

Conflict of interest: The authors have declared that no conflict of interest exists. Submitted: July 10, 2013; Accepted: July 3, 2014.

Reference information: J Clin Invest. 2014;124(9):3891-3900. doi:10.1172/JCI71946. before birth (4-6). Despite recent advances that have increased our knowledge of epidermal morphogenesis considerably, the function of the periderm remains poorly understood (7).

Recently, we have demonstrated that mice homozygous for a missense mutation in the transcription factor IFN regulatory factor 6 (IRF6), the gene mutated in the human congenital disorders Van der Woude syndrome and popliteal pterygium syndrome (8), exhibit a hyperproliferative epidermis that fails to undergo terminal differentiation, resulting in multiple interepithelial adhesions $(9,10)$. This phenotype is highly similar to that characterizing mice carrying mutations in either I $\mathrm{B}$ kinase- $\alpha(\mathrm{IKK} \alpha)$ or the cell-cycle regulator protein stratifin (SFN) (11-16). Despite these observations, which have been restricted to terminal differentiation stages of the epidermis or to abnormalities in specific tissues such as the developing secondary palate (10), detailed analyses of the early epidermal phenotype of $I r f 6^{R 84 C / R 84 C}, I k k a^{--}$, and $S \mathrm{fn}^{\mathrm{Er} / \mathrm{Er}}$ mice have not been undertaken.

In the current paper, we demonstrate that periderm is formed in a distinct pattern such that it encompasses the embryo at an early stage of development. We also show that periderm cells are highly polarized in their expression of adhesion complex proteins and are removed from the outer surface of the skin late in gestation by loss of contact with the newly formed cornified cells. Furthermore, by analyzing the early embryonic phenotype of $\operatorname{Irf6} 6^{R 84 C / R 84 C}, \mathrm{IkKa}^{-/}$, and $S f n^{E r / E r}$ mice and performing human genetic analyses, we demonstrate that failure of periderm development results in the abnor- 

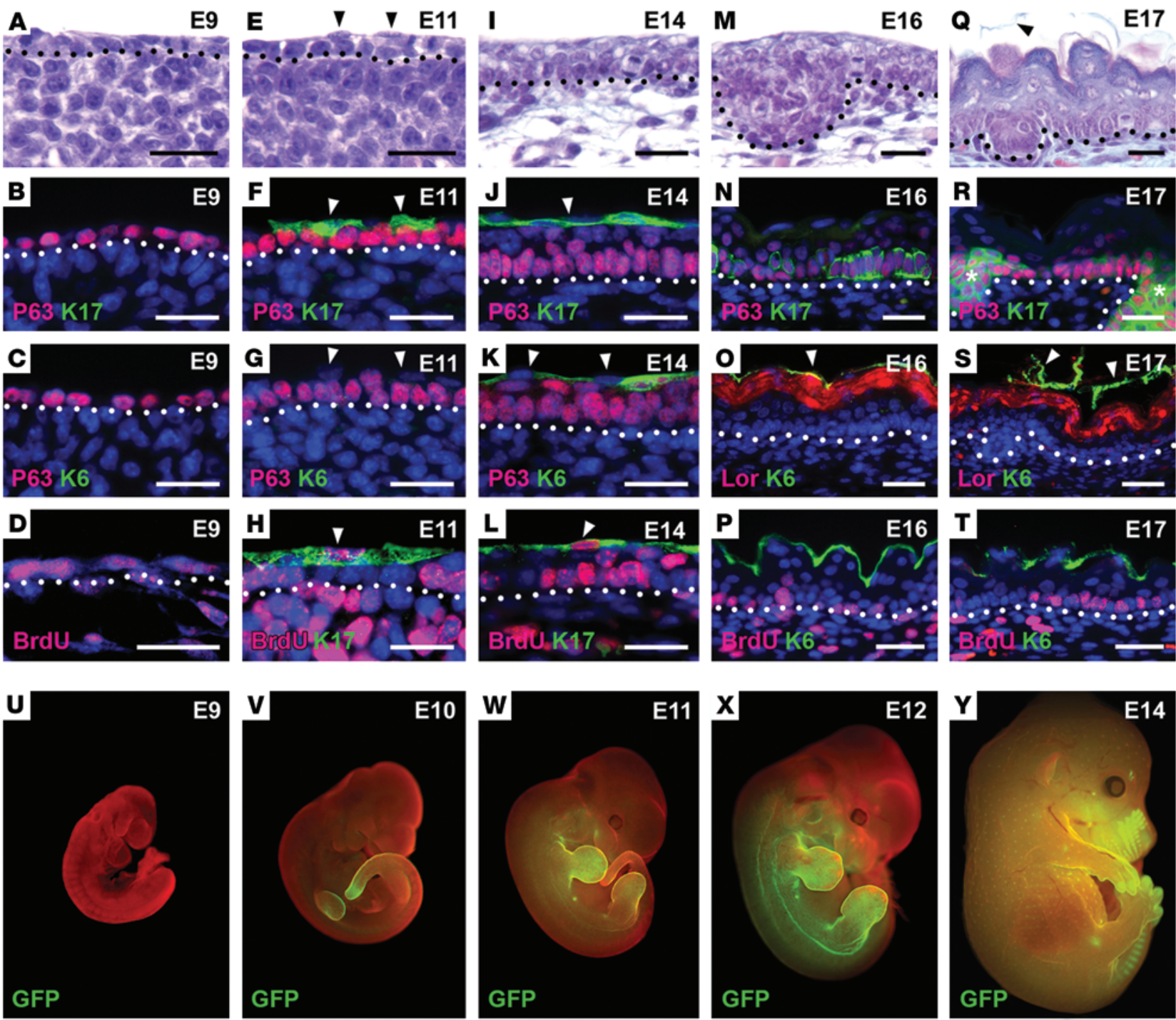

Figure 1. Periderm forms in a distinct pattern during embryogenesis. (A-D) E9 epidermis consists of a single layer of p63-positive, proliferative, cuboidal cells. (E-H) After E9, a single layer of keratin 17-positive, keratin 6-negative, proliferative, periderm cells (arrowheads) forms over the basal layer. (I-L) As further stratification of the epidermis proceeds and terminal differentiation commences; keratin 17- and keratin 6-positive periderm cells persist on the outermost surface (arrowheads). Proliferative periderm cells are still observed, but at a lower frequency than at earlier stages (L). (M-T) As terminal differentiation continues, keratin 17 expression is lost in the periderm, although it persists in developing hair follicles ( $\mathbf{N}$ and $\mathbf{R}$, asterisks). Keratin 6 persists as a marker of periderm at these later developmental stages ( $\mathbf{O}$ and $\mathbf{S}$ ). As the cornified layer forms, periderm detaches from the epidermal surface (arrowheads in $\mathbf{Q}$ and $\mathbf{S}$ ). At later developmental stages, proliferation is restricted to the lower epidermal layers ( $\mathbf{P}$ and $\mathbf{T})$. (U-Y) Fluorescence visualization of $[m K 17$ 5']-GFP transgenic mice reveals that periderm (green) forms in a distinct pattern during embryogenesis. Although absent at E9, periderm forms first over the developing tail and forelimb buds before spreading in a wave over the trunk and onto the head such that the embryo is covered in periderm by E14. Embryos were counterstained with propidium iodide (red). Dotted lines indicate the position of the basement membrane. Scale bars: $25 \mu \mathrm{m}$.

mal interepithelial adhesions that characterize the severe congenital disorders popliteal pterygium syndrome, cocoon syndrome, and Bartsocas-Papas syndrome.

\section{Results}

Periderm forms in a specific pattern. Despite recent advances that have increased our knowledge of epidermal morphogenesis, the function of periderm remains unknown (7). To provide an overview of the periderm life cycle, we analyzed the developing murine epidermis (Figure 1 and Supplemental Figure 1; supplemental material available online with this article; doi:10.1172/
JCI71946DS1). During E9, prior to periderm formation, the epidermis consisted of a single layer of proliferative, p63-positive cuboidal cells (Figure 1, A-D, Supplemental Figure 1, and ref. 17). An initial stratification event subsequently resulted in formation of the proliferative, keratin 17-positive periderm above the basal cells (Figure 1, E-H, and Supplemental Figure 1). Further stratification, commencing at E13, resulted in formation of an intermediate, keratin 1-expressing proliferative layer between the basal cells and the keratin 17-positive periderm; keratin 6 also marked the periderm from this age (Figure 1, I-L, Supplemental Figure 1, C, D, and F, and ref. 18). From approximately E15, 

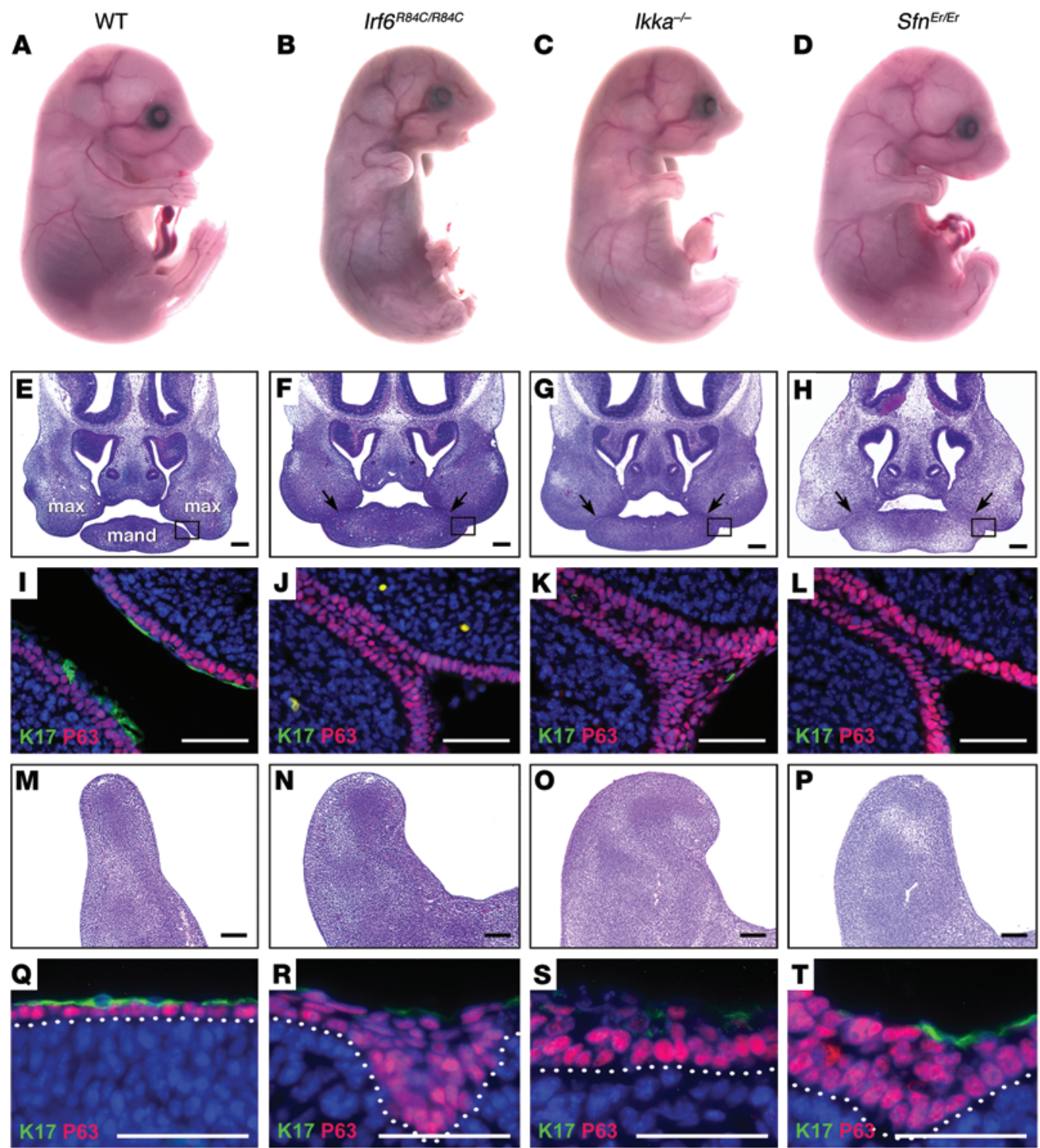

Figure 2. Irf6 ${ }^{R 84 C / R 84 C}, I k^{-1-}$, and $S f n^{E / E r}$ mice exhibit epithelial adhesions resulting from failure of periderm formation. (A-D) Gross morphological analysis of E16 mice reveals a highly similar phenotype in all 3 mutant strains. (E) Histological analysis of the facial complex of E12 wild-type mice reveals clearly separated maxillary and mandibular processes, whereas all mutant embryos (F-H) display abnormal maxillary-mandibular adhesions (arrows). (I-L) Immunofluorescence analysis of the regions of the facial complex boxed in $\mathbf{E}-\mathbf{H}$ reveals keratin 17-positive periderm cells (green) overlying p63-positive basal cells (red) (I). (J-L) However, in all 3 mutant strains, absence of keratin 17-positive periderm cells allows abnormal fusion between apposed p63-positive basal cells. (M-P) Transverse histological sections through hind limbs of E12 embryos reveals shortened curved limbs in all 3 mutant strains compared with wild-type littermates. (Q-T) Immunofluorescence analysis of keratin 17 (green) and p63 (red) reveals a continuous layer of keratin 17-positive periderm cells (arrowheads) overlying a layer of P63-positive basal cells in the epidermis of E12 wild-type mice (Q). (R-T) Similar analyses of mutant embryos reveal a thickened, disorganized basal layer with all cells expressing p63. Limited, weak expression of keratin 17 is observed in the epidermis of $I \mathrm{rfG}^{R 84 C / R 84 C}$ and $/ \mathrm{kka}^{-/-}$embryos compared with wild-type littermates (R and $\mathbf{S}$ ). Patchy expression of keratin 17 is occasionally observed on the outermost surface of the epidermis of $S f^{E r / E r}$ embryos (T). max, maxilla; mand, mandible. Scale bars: $100 \mu \mathrm{m}(\mathbf{E}-\mathbf{H} ; \mathbf{M}-\mathbf{P}) ; 50 \mu \mathrm{m}(\mathbf{I}-\mathbf{L} ; \mathbf{Q}-\mathbf{T})$.

keratin 17 expression was downregulated in the periderm and keratin 6 became a better marker for these cells (Figure 1, $\mathrm{N}$ and O, and Supplemental Figure 1, C and D). During E16, the epidermis began the terminal differentiation program with formation of the loricrin-positive granular layer above which a continuous layer of keratin 6-positive periderm cells was observed (Figure 1, $\mathrm{M}-\mathrm{O}$, and Supplemental Figure $1 \mathrm{G}$ ); however, when the outermost cornified layer had formed, the keratin 6-positive periderm detached from the epidermal surface (Figure 1, Q-S). Analyses of epidermal proliferation via BrdU incorporation and phosphohistone H3 immunofluorescence revealed multiple labeled cells within the basal and periderm layers (Figure 1, D, H, L, P, and T, and Supplemental Figure 1E). Quantification of the percentage of BrdU-positive cells during early epidermal development demonstrated a constant rate of proliferation within the basal layer, whereas the number of cell divisions within the periderm layer significantly reduced as differentiation of the underlying epidermis progressed (Supplemental Figure 2). 
E12

A
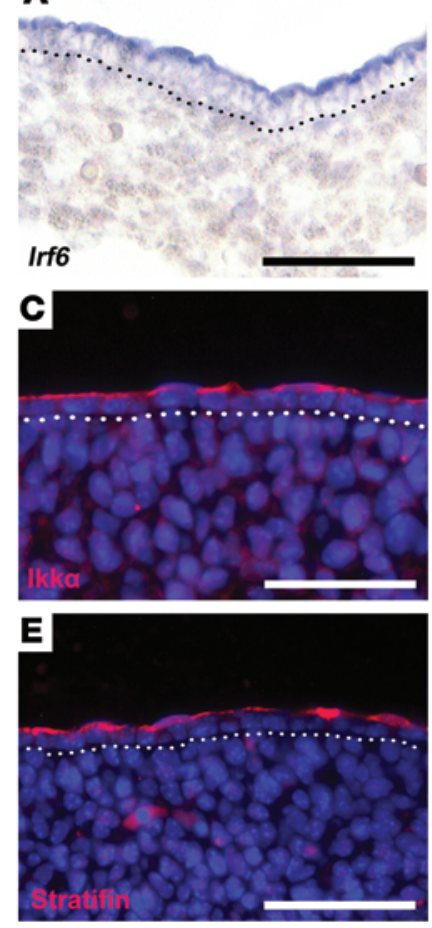

E14

B
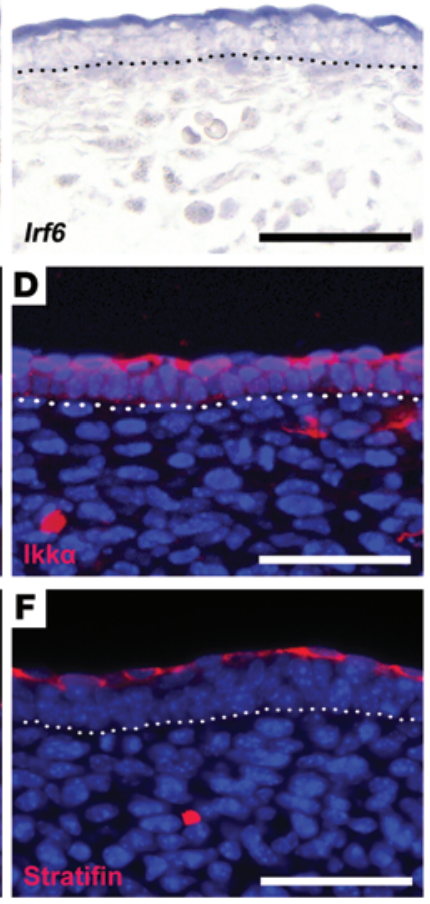

Figure 3. Irf6, IKK $\alpha$, and SFN are expressed in periderm early in epidermal development. (A and B) Section in situ hybridization reveals expression of Irf6 in the periderm of wild-type embryos at E12, with a similar pattern being observed in the multilayered epidermis at E14. (C and D) Immunofluorescence analyses reveal expression of I $k \mathrm{ka}$ in the periderm of wild-type embryos at E12 and E14. (E and F) Similarly, expression of Sfn is observed exclusively in the periderm of the developing epidermis of wildtype embryos at E12 and E14. The dotted line indicates the position of the basement membrane. Scale bars: $50 \mu \mathrm{m}$.

To visualize the pattern of periderm formation over the developing embryo in whole mount, we used [ $\left.m K 175^{\prime}\right]$-GFP transgenic mice in which GFP expression is driven by the keratin 17 promoter (Figure 1, U-Y, and Supplemental Figure 1). Expression of GFP in these mice recapitulates endogenous keratin 17 expression during embryogenesis (ref. 19 and Supplemental Figure 1, A-C). While no expression of GFP was observed in E9 [mK17 $\left.5^{\prime}\right]$-GFP transgenic embryos (Figure 1U), GFP expression was detected over the developing tail and forelimbs at E10 (Figure 1V). Subsequently, GFP expression was observed in the fore- and hind limb buds and spreading over the trunk and developing facial complex (Figure 1W). By E12, expression of GFP was almost continuous over the developing limbs, trunk, and facial complex (Figure 1X) such that GFP expression was observed over the entire body of [ $\left.m K 175^{\prime}\right]$-GFP transgenic mice by E14 (Figure 1Y). From approximately E15, keratin 17 expression was reduced in periderm cells and was induced in the embryonic ectoderm that is recruited for placode formation (Figure 1, N, R, Y, Supplemental Figure 1, A-C, and refs. 19, 20).

We have previously suggested a functional role for periderm within the oral cavity (10); however, detailed characterization of the life cycle of this layer has not been performed. Therefore, we carried out similar analyses of oral epithelial periderm formation (Supplemental Figure 3). Similar to the epidermis, oral periderm formed in a patterned manner, initially over the developing facial complex at E10.5 and later over the secondary palate (Supplemental Figure 3A). From E12.5, strong expression was also observed in the developing tooth germs. Immunofluorescence analysis of the oral epithelium revealed a delay in stratification when compared with the epidermis, with proliferative, keratin 17-positive periderm cells being observed only after E10.5 (Supplemental Figure 3, A-C and $\mathrm{E}$ ). As differentiation and stratification continued, proliferation and expression of keratin 1, E-cadherin, and loricrin was similar to that observed during epidermal development. At E12-E14, expression of p63 and keratin 6 and/or 17 expression remained mutually exclusive, whereas keratins 6 and 17 persisted in the intermediate layers of the oral epithelium from E16, with some cells additionally expressing p63 (Supplemental Figure 3, B-G).

$\operatorname{Irf6}{ }^{R 84 C / R 84 C}, \mathrm{Ikka}^{-/}$, and $\mathrm{Sfn}{ }^{\mathrm{Er} / \mathrm{Er}}$ mice lack periderm formation. We noted that mice carrying loss-of-function mutations in the transcription factor IRF6, the NF- $\kappa \mathrm{B}$ pathway component IKK $\alpha$, and the cellcycle regulator protein SFN exhibit highly similar defects of stratified, squamous, keratinizing epithelia, with the epidermis failing to undergo a normal differentiation program, resulting in absence of the granular and cornified layers. Consequently, multiple interepithelial adhesions among the hind limbs, tail, and body wall were observed (Figure 2, A-D, and refs. 9-16, 21). We analyzed the early epidermal phenotype in the corresponding mutant mice and demonstrated that interepithelial adhesions were present prior to the commencement of terminal differentiation of the epidermis (Figure 2, $\mathrm{E}-\mathrm{T}$ ). At E12, histological analysis of the facial complex revealed clearly separated maxillary and mandibular processes in wild-type embryos (Figure 2E), with keratin 17-positive periderm cells overlying p63-positive basal cells (Figure 2I). In contrast, $\operatorname{Irf6} 6^{R 84 C / R 84 C}$, $I k k a^{-/}$, and $S f n^{E r / E r}$ embryos exhibited abnormal adhesions between the epithelia covering these regions (Figure 2, F-H), which were devoid of keratin 17 expression (Figure 2, J-L). Transverse histological sections through E12 hind limbs revealed abnormal, shortened, curved limbs in all 3 mutant strains (Figure 2, N-P) compared with their wild-type littermates (Figure $2 \mathrm{M}$ ). While expression of $\mathrm{p} 63$ and keratin 17 was mutually exclusive in the epidermis of wild-type mice, consistent with a distinct p63-positive layer of basal cells covered by keratin 17-positive periderm cells (Figure 2Q), keratin 17 expression was markedly reduced in $I r f 6^{R 84 C / R 84 C}, I k \mathrm{ka}^{-/}$, and $S f n^{\mathrm{Er} / \mathrm{Er}}$ embryos, with any residual expression appearing patchy (Figure 2, $\mathrm{R}-\mathrm{T}$ ). Indeed, quantification of the area of keratin 17-positive immunofluorescence in the epidermis demonstrated a significant reduction in $\operatorname{Irf} 6^{R 84 C / R 84 C}$ embryos at E11 and E12 when compared with wild-type littermates (Supplemental Figure 4A).

Irf6, Ikka, and Sfn are expressed in the periderm during ectodermal development. Due to the epithelial adhesions observed from E11, we analyzed the expression of Irf6, IKK $\alpha$, and SFN at earlier stages of epidermal development. In situ hybridization (Figure 3, $\mathrm{A}$ and $\mathrm{B}$ ) and immunofluorescence (Figure 3, C-F) revealed that in the E12 epidermis (Figure 3, A, C, and E), when the epidermis was bilayered, and at E14, when an intermediate layer had formed (Figure 3, B, D, and F), expression of all 3 genes was observed predominantly in the periderm cells.

Periderm exhibits highly polarized expression of adhesion complex components. Given the abnormal epithelial adhesions observed in $\operatorname{Irf6} 6^{R 84 C / R 84 C}, I k k a^{-/}$, and $S f n^{E r / E r}$ embryos, we analyzed their early 
WT
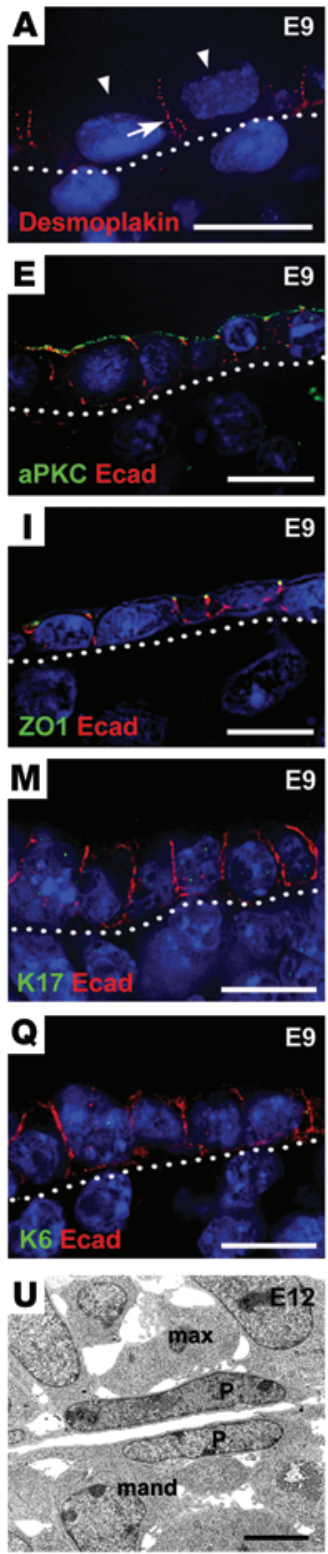

IIf6 $6^{\text {R84C/R84C }}$
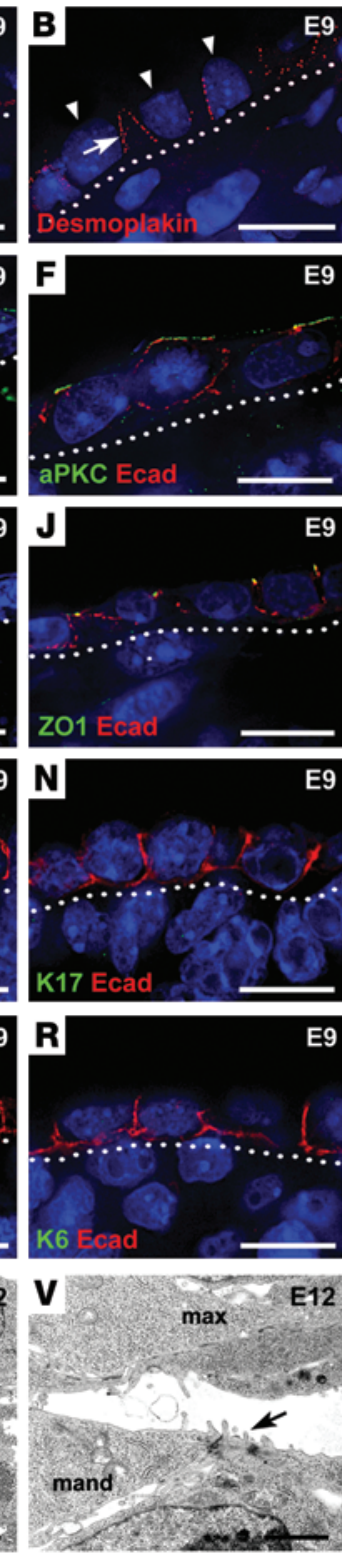

WT
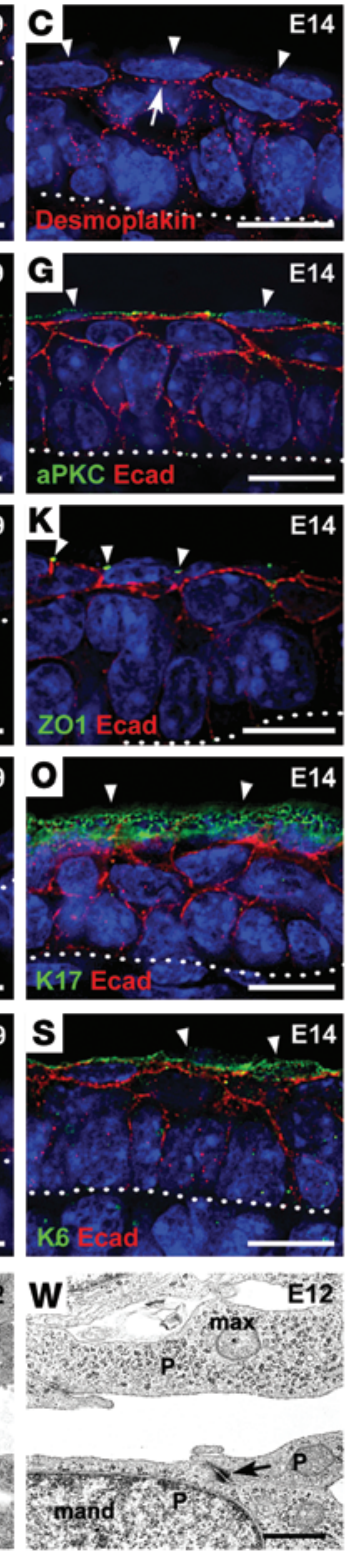
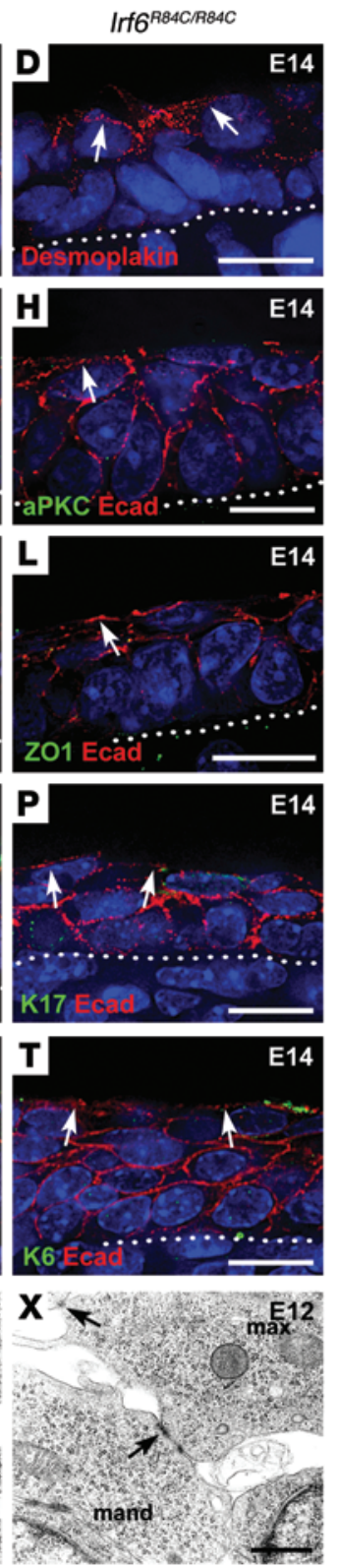

Figure 4. Periderm cells are highly polarized in their expression of adhesion complex proteins. (A-D) Deconvolution analysis indicates that in E9 wild-type and Irf6 ${ }^{R 84 C / R 84 C}$ mice, desmoplakin is expressed between basal cells (arrows) but is absent from their apical surface (arrowheads). At E14, while absent from the apical surface of wild-type epidermis (arrowheads), desmoplakin is expressed apically in Irf6 $6^{R 84 C / R 84 C}$ embryos (arrows). (E-L) Expression of aPKC and ZO1 together with E-cadherin demonstrates that periderm cells are highly polarized, preventing adhesion complex protein expression on the apical surfaces of wild-type epidermis, whereas loss of these cells in Irf6 ${ }^{R 84 / R 84 C}$ embryos results in E-cadherin expression apically (arrows). (M-T) Additional analysis of E-cadherin together with the periderm markers keratin $17(\mathbf{M}-\mathbf{P})$ and keratin $6(\mathbf{Q}-\mathbf{T})$ confirms the absence of this layer at E9 and continued absence of periderm at E14 in mutant epidermis ( $\mathbf{P}$ and $\mathbf{T})$. (U-X) TEM indicates that, in contrast with wild-type mice, the oral epithelia of E12 Irf6 $6^{R 4 C / R 84 C}$ littermates lack periderm cells and exhibit multiple apical protrusions on the outer surfaces (arrow in $\mathbf{V}$ ). Higher magnification demonstrates that desmosomes connect adjacent periderm cells in wild-type mice (arrow in W), but are absent from their apical surfaces; in contrast, desmosomes are clearly visible between the oral surfaces of apposed epithelia in regions of abnormal epithelial adhesion in Irf6 ${ }^{R 84 / R 84 C}$ embryos (arrows in $\mathbf{X}$ ). p, periderm. Scale bars: $100 \mu \mathrm{m}(\mathbf{A}-\mathbf{T}) ; 3 \mu \mathrm{m}$ (U and $\mathbf{V}$ ); $600 \mathrm{~nm}(\mathbf{W}$ and $\mathbf{X})$.

and the apical polarity marker atypical PKC (aPKC) (22), in all 3 mutant strains (Figure 4 and Supplemental Figure 5). At E9, prior to periderm formation, punctate distribution of desmoplakin was observed between adjacent cells of the ectodermal monolayer, but was absent from their apical surfaces in both wild-type and mutant embryos (Figure 4, A and B). Dual analysis of E-cadherin and aPKC confirmed the absence of adhesion complex components along the apical surface of the epidermis of all E9 embryos, when this region exhibited strong aPKC expression (Figure 4, E and F, and Supplemental Figure 5, A-D). Similarly, prior to periderm formation, wild-type and mutant embryos displayed punctate expression of $\mathrm{ZO} 1$ directly above E-cadherin staining at the apical-most borders of the lateral surface between the single-layered epidermal cells (Figure 4, I and J, and Supplemental Figure 5, E-H). As expected, keratin 17 and keratin 6 expression were absent from the preperiderm epidermis of wild-type and mutant mice (Figure $4, \mathrm{M}, \mathrm{N}, \mathrm{Q}$, and R).

These data demonstrate thatprior toperidermformation, the epidermis of $\operatorname{Irf6} 6^{R 84 C / R 84 C}$, $I k k^{-/-}$, and $S n^{E r / E r}$ embryos resembles that of their wildtype littermates. Subsequently, we performed the same analysis on older embryos (Figure 4 and Supplemental Figure 5). At E14, when the epidermis consists of an inner basal layer, 1 or 2 layers of suprabasal cells, and an outer layer of periderm, desmoplakin expression was observed between the basal cells, around the suprabasal cells, and on the basolateral surfaces of the periderm cells in wild-type epidermal development in greater detail by investigating the distribution of the adhesion molecules desmoplakin, an obligate component of desmosomes, and E-cadherin, an essential component of epithelial adherens junctions, the tight junction component ZO1, embryos, while the apical surfaces of periderm cells were devoid of desmoplakin expression (Figure 4C); in contrast, expression of desmoplakin was clearly visible on the outer surface of the epidermis of the mutant embryos (Figure 4D). Similarly, expression of 


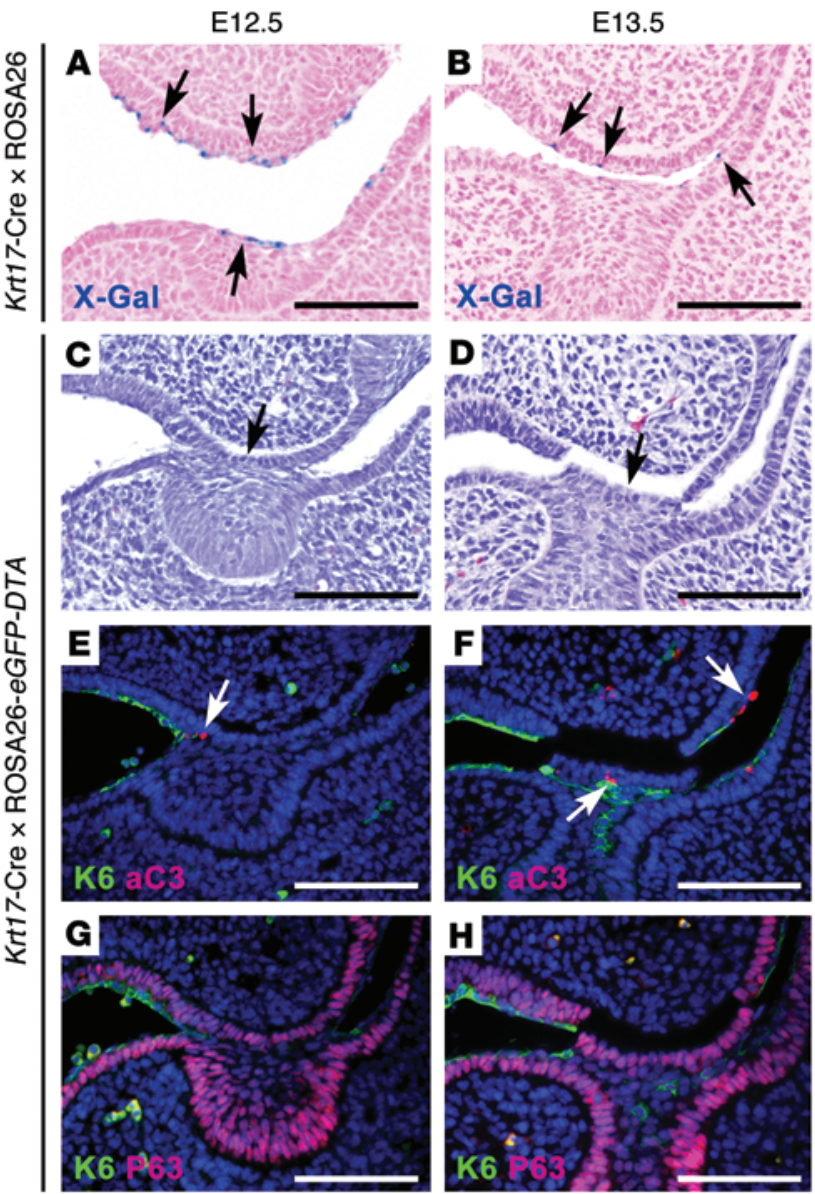

E-cadherin was observed around the basal and intermediate cells, but was absent from the apical surfaces of wild-type periderm cells, which exhibited strong aPKC expression (Figure 4G and Supplemental Figure 5I); ZO1 expression was observed between the periderm cells (Figure $4 \mathrm{~K}$ and Supplemental Figure 5M). In striking contrast, failure of highly polarized periderm formation resulted in expression of adhesion complex proteins on the apical surfaces of the exposed intermediate cells in E14 Irf6 ${ }^{R 84 C / R 84 C}$, I $k_{k a /}$, and $S f n^{E r / E r}$ embryos (Figure 4, H and L, and Supplemental Figure $5, \mathrm{~J}-\mathrm{L}$, and $\mathrm{N}-\mathrm{P}$ ). Analysis of the periderm markers keratin 17 and keratin 6, with E-cadherin confirmed the loss of these markers in mutant embryos where intermediate cells were exposed, consistent with the loss of the periderm cells (Figure 4, O, P, S, and T).

Due to the similarities in epidermal and oral epithelial periderm formation and the observed epithelial adhesions at both of these sites in all 3 mutant mouse strains, we analyzed affected oral epithelia. Ultrastructural examination of $\operatorname{Irf} 6^{\text {R84C/R84C }}$ embryos confirmed the absence of periderm on the oral epithelia surface (Figure $4, \mathrm{~V}$ and $\mathrm{X}$ ). In areas where adjacent epithelia were in direct contact, transmission electron microscopy (TEM) analysis revealed periderm overlying the basal cells in wild-type embryos at E12 (Figure $4 \mathrm{U}$ ); however, in Irf6 ${ }^{R 84 C / R 84 C}$ littermates, periderm was absent and multiple apical protrusions were visible projecting into the cavity between the adjacent maxillary and mandibular epithelia (Figure $4 \mathrm{~V}$ ). Quantification of these apical protrusions revealed a significant increase in $\operatorname{Irf} 6^{R 84 / R 84 C}$ embryos at E11 and
Figure 5. Genetic ablation of periderm results in intraoral fusions. ( $A$ and B) X-gal staining of Krt17-Cre $\times$ ROSA26 bitransgenic mice indicates that Cre-mediated excision is restricted to the intraoral periderm (arrows). (C and D) Histological analysis of Krt17-Cre $\times$ ROSA26-eGFP-DTA bitransgenic embryos reveals adhesions between the maxillary and mandibular epithelia in regions of Cre-mediated excision. Separation of the epithelium from the underlying mesenchyme is observed in some regions (arrows). (E and $\mathbf{F}$ ) Activated caspase 3 staining identifies peridermal cell death resulting from $\mathrm{Krt17}$-Cre-directed DTA expression (arrows). (G and $\mathbf{H}$ ) Immunofluorescence analysis reveals that the periderm marker keratin 6 (green) is largely absent from regions where the juxtaposed epithelia have adhered, but present in regions flanking the adhesions. P63 staining identifies basal cells (red). Scale bars: $100 \mu \mathrm{m}$.

E12 when compared with their wild-type littermates (Supplemental Figure 4B). At higher magnification, desmosomes were observed between adjacent periderm cells of wild-type embryos with a clear gap between the maxillary and mandibular surfaces (Figure $4 \mathrm{~W}$ ); in contrast, adhesion complexes were clearly visible connecting the oral surfaces of apposed epithelia in regions of abnormal epithelial adhesion in Irf ${ }^{R 84 C / R 84 C}$ embryos (Figure $4 \mathrm{X}$ ).

Specific ablation of periderm cells partially phenocopies mutant

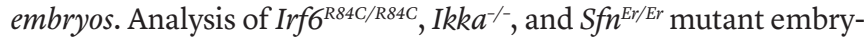
os strongly suggested that epidermal and oral epithelial periderm has highly polarized expression of adhesion complexes and plays a fundamental role in early ectodermal development by preventing intimately apposed, adhesion-competent epithelia from adhering to one another. To confirm this hypothesis, we harnessed an in vivo genetic ablation strategy that utilized Cre recombinase-mediated (Cre-mediated) expression of diphtheria toxin fragment A (DTA), which leads to cell death specifically in Cre-expressing cells with no nonspecific bystander effect, an approach that is independent of the underlying molecular pathways involved (23). To provide for periderm-specific expression of DTA during early embryonic development, we cloned the proximal promoter of the mouse keratin 17 gene (Krt17) upstream of a Cre-recombinase cassette. Intercrossing $\mathrm{Krt17}$-Cre transgenic mice with ROSA26 conditional mice confirmed that the pattern of Cre-mediated excision, although patchy, broadly recapitulated the expression of endogenous keratin 17 in the oral periderm, but not in the epidermis (Figure 5, A and B). Subsequently, the oral epithelia of embryos derived from crossing Krt17-Cre and ROSA26-eGFP-DTA mice were analyzed, with adhesions between the maxillary and mandibular epithelia being visible from E12.5 and persisting throughout development (Figure 5, C-H). As the $\mathrm{Krt17}$ promoter used is not expressed in the epithelium covering the dorsum of the tongue until close to birth (19), the adhesions were generally limited to the lateral regions of the oral cavity; however, in severe cases, they prevented normal development of the palatal shelves, resulting in cleft palate (Supplemental Figure 6). Immunofluorescence and TEM analyses indicated that periderm cells underwent caspase-mediated apoptosis and were absent in regions of interepithelial adhesion, allowing the formation of adhesion complexes between exposed basal or intermediary cells (Figure 5, E-H, and Supplemental Figure 7).

Mutation of IKKA results in failure of periderm formation during human embryogenesis. In humans, pterygium syndromes are complex congenital malformations that encompass several distinct but overlapping clinical conditions characterized by epidermal 

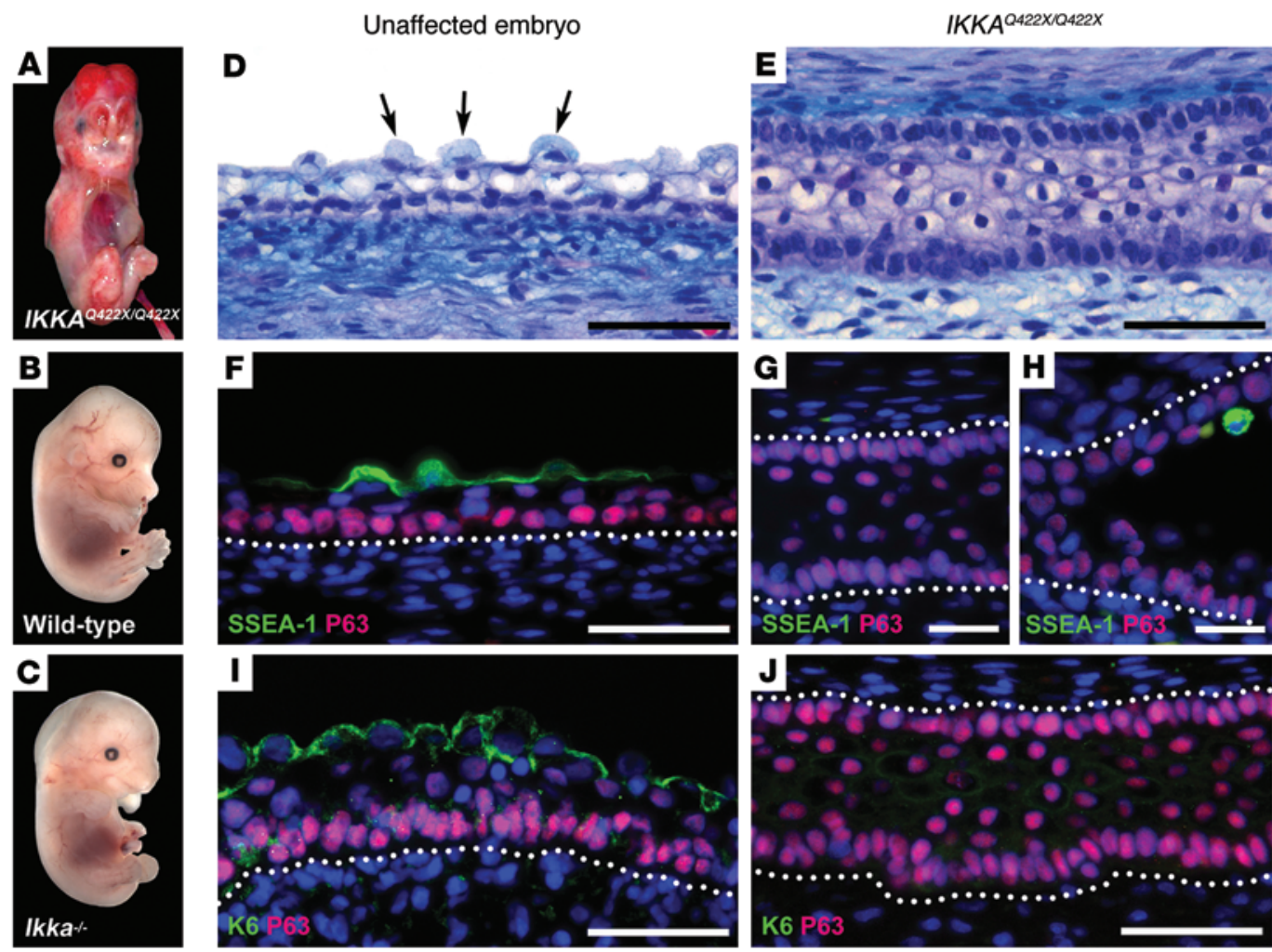

Figure 6. Failure of periderm formation results in pathological adhesions in humans. (A) Frontal view of a fetus with Cocoon syndrome illustrating the severe interepithelial adhesions. (B and C) Lateral views of E14 wild-type and $/ \mathrm{kk}^{-/-}$mice demonstrating the phenotypic overlap with Cocoon syndrome. (D and E) Histological analysis reveals that while a single layer of periderm cells is visible in the epidermis of a normal fetus (arrows), this layer is absent in Cocoon syndrome and apposed epithelial layers have adhered to one another. (F-H) Immunostaining confirms that SSEA-1-positive periderm cells (green) overlying a layer of p63-positive basal cells (red) are present in an unaffected fetus but absent in fused (C) and nonfused (H) epidermal regions in Cocoon syndrome. Only occasional SSEA-1-positive cells are observed in regions where the epidermal surfaces are unfused (H). (I and J) Immunostaining with keratin 6 antisera (green) confirms the absence of periderm on the surface of IKKA ${ }^{0422 x / 0422 x}$ epidermis. Nuclei are counterstained with DAPI (blue). Scale bars: $50 \mu \mathrm{m}$.

webbing and associated craniofacial anomalies. Notably, mutations in IRF6 and IKK $\alpha$ underlie popliteal pterygium syndrome and cocoon syndrome, respectively $(8,24)$. Recently, the homozygous nonsense mutation p.Gln422X in IKKA has been shown to underlie cocoon syndrome, a lethal autosomal recessive syndrome characterized by multiple malformations, most notably defective facial development and limbs that are bound to the trunk and encased under the skin, thereby phenocopying Ikka knockout mice (Figure 6, A-C, and ref. 24). In light of the above observations, we hypothesized that periderm development was disrupted in the affected fetus. Although keratin 6 marks periderm in human fetal skin, keratin 17 is present throughout the developing fetal epidermis, so an alternative, periderm-specific marker was utilized. Histological analysis and immunostaining with keratin 6 and stage-specific embryonic antigen-1 (SSEA-1) antisera, which are periderm specific in the developing human fetal epidermis (25-27), confirmed the absence of epidermal and oral periderm formation in the affected fetus, mirroring the observations made in $\mathrm{Ikka}^{--}$mice (Figure 6, D-J, and Figure 2K).

\section{Discussion}

Appropriate development of stratified, squamous, keratinizing epithelia generates an outer protective permeability barrier that prevents water loss, entry of toxins, and microbial invasion. Dur- ing embryogenesis, the first stratification event of the immature ectoderm produces a single layer of undifferentiated, cuboidal epithelial cells that stratifies to produce an outer layer of flattened periderm cells. Although several functions have been proposed for the periderm, including protection from the uterine environment (28), regulation of underlying mesenchyme (29), and contribution to cornified envelope formation (30), its life cycle and function(s) remain largely unexplored.

In the current paper, we have used [ $\left.m K 175^{\prime}\right]$-GFP transgenic mice to delineate the formation of periderm in whole mount. Our results indicate that periderm forms in a specific and reproducible pattern during E10-E12, with this cell layer being observed first over the limbs and tail before subsequently spreading in a wave over the face and torso. After its formation, periderm persists throughout development as a single-cell layer until it is removed from the outer surface of the terminally differentiating epidermis as it acquires barrier function during E16-E17. Barrier formation is patterned, arising at conserved initiation sites on the dorsum and head before spreading across the epidermis as a moving front converging at the dorsal and ventral midlines; in the mouse, periderm is lost in a patterned manner that recapitulates barrier formation (31).

Prior to periderm formation, the single layer of cells that make up the developing ectoderm is connected via desmosomes and adherens junctions, expression of which is delimited by ZO1-pos- 
itive tight junctions. In contrast, the apical surfaces of these cells express the apical polarity marker aPKC and are devoid of expression of adhesion complex proteins. These observations suggest that, before the periderm forms, the ectodermal cells are incapable of adhering to adjacent ectodermal surfaces. With continuing development, the naive ectoderm progresses through a defined series of stratification and differentiation events to produce the mature epidermis, during which the constituent cells maintain cell-cell attachments on all surfaces to preserve the integrity of the multilayered structure. Notably, defects in cell-cell connectivity result in a wide variety of pathologies, including epidermolysis bullosa, ectodermal dysplasia, and epidermal blistering (32).

As embryonic development progresses, the apical surfaces of the periderm cells remain devoid of desmoplakin and E-cadherin expression. The presence of ZO1-positive tight junctions, which demarcate the boundary between apical and basolateral surfaces of the periderm cells, appears to provide a "fence function" whereby they act as a barrier to prevent spread of adhesion complexes onto the apical surface (22). This highly polarized expression of adhesion complexes in periderm cells suggested that periderm acts as a protective barrier that prevents pathological adhesion between intimately apposed, adhesion-competent epithelia. To test this hypothesis, we analyzed a series of mutant mice that exhibit a highly similar phenotype characterized by multiple soft tissue adhesions that result in the hind limbs, tail, and body wall being fused and the oral cavity occluded.

Previously, we and others have shown that mice carrying mutations in IRF6, IKK $\alpha$, and SFN exhibit abnormalities of late-stage epidermal/oral epithelial development in which the keratinocyte proliferation/differentiation switch is disturbed, resulting in a hyperproliferative epidermis that fails to undergo terminal differentiation $(9-16,21)$. Despite these observations, detailed analyses of the onset of these abnormalities have not been undertaken. Here, we have demonstrated that all 3 mutant strains exhibit a subtle but highly reproducible phenotype during early embryogenesis that is characterized by epithelial adhesions between the maxillary and mandibular processes of the developing facial complex and on the ventral surface of the developing limb buds. Notably, all 3 mutant strains fail to develop normal periderm, with multiple membrane protrusions forming on the apical surface of the exposed basal cells. These observations are reminiscent of the morphological events observed during dorsal closure in Drosophila embryos and in wound closure $(33,34)$. Together, these findings indicate that the presence of periderm prevents exposed, adhesion-competent, basal cells from actively contacting one another: in essence, periderm acts as a protective "Teflon" coat. In addition to this mechanical protective function of the periderm cells, we cannot exclude the possibility that this layer may also contribute to the signaling control of epidermal development and differentiation. This possibility is supported by the expression of Irf6, IKK $\alpha$, and SFN specifically in the periderm cells long after formation of this layer and secondary stratification of the underlying epidermal cells (e.g., E14; Figure 3, B, D, and F). Additionally, abnormal epidermal differentiation was observed in mutant embryos as early as E12, as demonstrated by expansion of p63-expressing cell layers. Further experiments will be required to determine whether this is a specific consequence of the loss of the individual gene expression or due to a global loss of periderm cells.
Although we have assigned an embryonic barrier function to periderm, the molecular events driving formation of this cell layer remain only partially characterized. While IRF6, IKK $\alpha$, and SFN clearly play a fundamental role in periderm formation, it is not clear how these proteins interact with each other and with additional components of the underlying gene regulatory network. IRF6 is a transcription factor characterized by a highly conserved pentatryptophan DNA-binding domain and a less well-conserved protein interaction domain that binds to an IFNstimulated response element similar to that to which other IRF family members bind $(35,36)$. IRF6 has recently been shown to promote differentiation of zebrafish periderm by directly regulating the expression of Grainyhead-like 3 (GRHL3) (37). Despite these observations, subcellular localization studies indicate that IRF6 localizes to the cytoplasm both in vivo and in vitro $(9,21$, 38,39 ) suggesting that, as with other IRF family members, IRF6 exists in an autoinhibited state until activated by phosphorylation (40). Although previous studies have indicated that IRF6 is subject to phosphorylation, the kinase responsible has not been identified. While IKK $\alpha$ is critical for normal differentiation of epidermal keratinocytes, this function is independent of its kinase function (12-14, 41). In contrast, the observations that Ripk4-null mice phenocopy the mutant strains analyzed in this study (42), while mutations in RIPK4 underlie Bartsocas-Papas syndrome $(43,44)$, which is characterized by multiple skin webs affecting the flexural surfaces, often accompanied by craniofacial anomalies, suggest that this molecule is a candidate for the kinase responsible for IRF6 phosphorylation.

Although the kinase function of IKK $\alpha$ is dispensable for keratinocyte differentiation, SFN is downregulated in IKK $\alpha$-deficient keratinocytes, with reintroduction of IKK $\alpha$ into IKK $\alpha$-deficient keratinocytes restoring SFN expression (45). Although we have been unable to establish a biochemical interaction between IRF6 and SFN/IKK $\alpha$, we have demonstrated an epistatic interaction between Irf6 and Sfn (9). Notably, SFN has been shown to bind phosphorylated keratin 17 , an interaction which is critical for cytoplasmic retention of SFN, promotion of Akt/mTOR signaling, protein synthesis, and cell growth in keratin 17-deficient keratinocytes (46). The coexpression of SFN and keratin 17 in the periderm raises the possibility that, as in hair follicles of normal skin and in wounded epithelial cells, these proteins interact in the control of protein synthesis and cell growth of periderm cells.

In summary, we have demonstrated that periderm cells form in a distinct and reproducible pattern early in embryogenesis, exhibit highly polarized expression of adhesion complexes, and are shed from the outer surface of the skin late in development as the epidermis acquires its barrier function. Moreover, we show that the periderm plays a transient but fundamental role during embryogenesis by preventing pathological adhesion between intimately apposed, adhesion-competent epithelia and that disruption of the protective barrier function of periderm underlies a series of birth defects that exhibit multiple interepithelial adhesions, including the autosomal dominant popliteal pterygium syndrome and the autosomal recessive conditions cocoon syndrome and BartsocasPapas syndrome. Interestingly, the recent observation that dominant mutations in GRHL3 cause Van der Woude syndrome and disrupt oral periderm development (47) supports the protective 
barrier function of periderm and widens the clinical conditions that can be classed as "peridermopathies."

\section{Methods}

Generation of mutant mice. Generation and maintenance of mice carrying the targeted $I r f \sigma^{+/ R 84 C}$ and $I k k a^{+/}$alleles and the radiation-induced $S \mathrm{fn}^{+/ E r}$ allele have been described previously $(9,11,12)$. The mouse Krt17 promoter was PCR-amplified using the forward primer: $5^{\prime}-($ Not $\mathrm{I})$ TTGTCCTGTCATTGGCTCAG-3' and the reverse primer: $5^{\prime}$-(EcoRI) CATGGTGGCAGCGGGCAA- $3^{\prime}$ and cloned into the pBS CreES vector, which is based on pBluescript II KS+ but contains the Cre recombinase coding sequence attached to a nuclear localization signal (a gift from R. Boot-Handford, University of Manchester). Linear DNA containing just the Krt17 promoter and the Cre recombinase coding sequence were sent to the Transgenic Mouse Facility (Center of Functional Genomics, University at Albany, State University of New York, Rensselaer, New York, USA) for pronuclear injection. Founder Krt17-Cre mice were bred with C57BL/6 wild-type mice to generate transgenic offspring.

Histological, section in situ hybridization, and immunofluorescence analyses. For histological analysis, embryos were dissected from timemated pregnant female mice, the morning on which the vaginal plug was detected being designated E0.5. Embryos were staged on the basis of external morphological characteristics, fixed in Bouin's solution, dehydrated through a graded ethanol series, cleared in chloroform, embedded in wax, sectioned at $6 \mu \mathrm{m}$, and stained with H\&E. Nonradioactive section in situ hybridization was performed as described previously (48), with the exception that sections were detected using BM Purple (Roche). The Irf6 probe has been described previously (49). Detection of $\beta$-galactosidase on frozen sections was performed as described previously (50). For immunofluorescence analyses, $4 \%$ paraformaldehyde-fixed sections were treated with $10 \mathrm{mM}$ citrate buffer at $96^{\circ} \mathrm{C}$ for 10 minutes for antigen retrieval. Sections were incubated overnight at $4^{\circ} \mathrm{C}$ with antibodies against the following: p63 (4A4; 1:50; Santa Cruz Biotechnology Inc.); IKK $\alpha$ (1:100; Imgenex Corp.); SFN (C-18; 1:20; Santa Cruz Biotechnology Inc.); keratin 17 (1:1000); keratin 6 (1:500; Covance Research Products); loricrin (1:500; Covance Research Products); keratin 1 (1:200; Covance Research Products); E-cadherin (1:200; BD Biosciences); desmoplakin (11-5F; 1:10; a gift from D. Garrod, University of Manchester); activated caspase 3 (1:750; R \& D Systems); ZO-1 (1:400; Life Technologies); GFP (1:800; Life Technologies); histone H3 (S10; 1:1000; Abcam); aPKC (C-20; 1:400; Santa Cruz Biotechnology Inc.); and SSEA-1 (1:1). The monoclonal antibody SSEA-1, developed by D. Solter, was obtained from the Developmental Studies Hybridoma Bank, developed under the auspices of the National Institute of Child Health and Human Development (NICHD) and maintained by the University of Iowa, Department of Biological Sciences (Iowa City, Iowa, USA). Proliferation assays were performed by intraperitoneal injection of BrdU-labeling reagent $(100 \mu \mathrm{g} / \mathrm{g}$ body weight) into pregnant mice, which were sacrificed after 2 hours. Embryos were processed as above and immunostained with an anti-BrdU antibody (1:100; Abcam). Sections were counterstained with DAPI and visualized using a Leica DMRB microscope. For deconvolution analysis, images were acquired on a Delta Vision RT restoration microscope (Applied Precision) using a $\times 60 / 1.40$ PlanApo objective and the Sedat filter set (Chroma 89000). Images were collected using a Coolsnap HQ camera (Photometrics) with a $\mathrm{Z}$ optical spacing of $0.2 \mu \mathrm{m}$. Raw images were deconvolved using Softworx software, and maximum intensity projections of these deconvolved images are shown in Figure 4 and Supplemental Figure 4. The area of keratin 17-positive immunofluorescence was determined using ImageJ software (http://imagej.nih. gov/ij/) to calculate the relative area of red fluorescence within a single maximum projection image. All analyses were performed on at least 3 mice for each genotype at each time point, except for those shown in Supplemental Figures 1 and 3, where analysis at each time point was performed on tissue from the same mouse.

TEM analysis. For TEM, small regions of skin were dissected from E12 embryos and fixed in 2\% paraformaldehyde/2\% glutaraldehyde in $0.1 \mathrm{M}$ sodium cacodylate buffer containing $0.15 \mathrm{M}$ sucrose and $2 \mathrm{mM}$ calcium chloride ( $\mathrm{pH}$ 7.3). Samples were washed with cacodylate buffer, post-fixed with $1 \%$ osmium tetroxide, dehydrated through a graded ethanol series, and embedded in agar 100 resin. Ultrathin sections were contrasted with uranyl acetate and lead citrate and examined on a Philips model 400 transmission electron microscope.

Statistics. Statistical significance was determined using an unpaired, 2-tailed Student's $t$ test. A $P$ value of less than 0.05 was considered significant.

Study approval. All animal experiments were approved by the University of Manchester Ethical Review Committee and performed in accordance with the Animals (Scientific Procedures) Act, 1986, United Kingdom. The fetus with Cocoon syndrome has been reported previously (24). The parents provided written informed consent to participate in this study, which was approved by the ethics committees of the Joint Authority for the Hospital District of Helsinki and Uusimaa, Finland. Tissue from a similarly aged unaffected fetus was obtained with approval from the North West Regional Ethics Committee (H1010/28), informed consent and guidelines of the Polkinghorne committee, and following United Kingdom Human Tissue Authority regulations. Five-micron sections were obtained from formalin-fixed, wax-embedded archived blocks and subject to histological staining or immunofluorescence as above.

\section{Acknowledgments}

We thank the family for participating in the research and Marjo Kestilä both for facilitating access to patient samples and for commenting on the manuscript. We thank Samantha Newby, Electron Microscopic Service, for her expert assistance with TEM, and the Bio-imaging Facility, University of Manchester, for assistance with the deconvolution analysis. We would also like to thank Steven Murray and Caleb Haffner for help with the Krt17-Cre experiments. N. Hanley is a Wellcome Trust Senior Fellow in clinical science. This research was supported financially by the Wellcome Trust (082868, 097820), the Medical Research Council (G0901539), the Healing Foundation, the NIH research-funded Manchester Biomedical Research Centre (to M.J. Dixon), and grant AR44232 from the NIH (to P.A. Coulombe).

Address correspondence to: Michael Dixon, Michael Smith Building, University of Manchester, Oxford Road, Manchester M13 9PT, United Kingdom. Phone: 44.0.161.275.5620; E-mail: mike. dixon@manchester.ac.uk.

Rebecca J. Richardson's present address is: Department of Physiology and Pharmacology, University of Bristol, Medical Sciences, Bristol, United Kingdom. 
1. Dotto GP. Signal transduction pathways controlling the switch between keratinocyte growth and differentiation. Crit Rev Oral Biol Med. 1999;10(4):442-457.

2. Fuchs E, Raghavan S. Getting under the skin of epidermal morphogenesis. Nat Rev Genet. 2002;3(3):199-209.

3. Watt FM. Stem cell fate and patterning in mammalian epidermis. Curr Opin Genet Dev. 2001;11(4):410-417.

4. M'Boneko V, Merker HJ. Development and morphology of the periderm of mouse embryos (days 9-12 of gestation). Acta Anat (Basel). 1988;133(4):325-336.

5. Holbrook KA, Odland GF. The fine structure of developing human epidermis: light, scanning, and transmission electron microscopy of the periderm. J Invest Dermatol. 1975;65(1):16-38.

6. Sanes JR, Rubenstein JL, Nicolas JF. Use of a recombinant retrovirus to study post-implantation cell lineage in mouse embryos. EMBO J. 1986;5(12):3133-3142.

7. Fuchs E. Scratching the surface of skin development. Nature. 2007;445(7130):834-842.

8. Kondo S, et al. Mutations in IRF6 cause Van der Woude and popliteal pterygium syndromes. Nat Genet. 2002;32(2):285-289.

9. Richardson RJ, et al. Irf6 is a key determinant of the keratinocyte proliferation-differentiation switch. Nat Genet. 2006;38(11):1329-1334.

10. Richardson RJ, Dixon J, Jiang R, Dixon MJ. Integration of IRF6 and Jagged2 signalling is essential for controlling palatal adhesion and fusion competence. Hum Mol Genet. 2009;18(14):2632-2642.

11. Guenet JL, Salzgeber B, Tassin MT. Repeated epilation: a genetic epidermal syndrome in mice. JHered.1979;70(2):90-94.

12. Hu Y, et al. Abnormal morphogenesis but intact IKK activation in mice lacking the IKK $\alpha$ subunit of IкB kinase. Science. 1999;284(5412):316-320.

13. Li Q, et al. IKK1-deficient mice exhibit abnormal development of skin and skeleton. Genes Dev. 1999;13(10):1322-1328.

14. Takeda K, et al. Limb and skin abnormalities in mice lacking IKKa. Science. 1999;284(5412):313-316.

15. Herron BJ, et al. A mutation in stratifin is responsible for the repeated epilation (Er) phenotype in mice. Nat Genet. 2005;37(11):1210-1212.

16. Li Q, Lu Q, Estepa G, Verma IM. Identification of 14-3-3sigma mutation causing cutaneous abnormality in repeated-epilation mutant mouse. Proc Natl Acad Sci U S A. 2005;102(44):15977-15982.

17. Byrne C, Tainsky M, Fuchs E. Programming gene expression in developing epidermis. Development.1994;120(9):2369-2383.

18. Lechler T, Fuchs E. Asymmetric cell divisions promote stratification and differentiation of mammalian skin. Nature. 2005;437(7056):275-280.

19. Bianchi N, Depianto D, McGowan K, Gu C, Coulombe PA. Exploiting the keratin 17 gene promot- er to visualize live cells in epithelial appendages of mice. Mol Cell Biol. 2005;25(16):7249-7259.

20. McGowan KM, Coulombe PA. Onset of keratin 17 expression coincides with the definition of major epithelial lineages during skin development. JCell Biol. 1998;143(2):469-486.

21. Ingraham CR, et al. Abnormal skin, limb and craniofacial morphogenesis in mice deficient for interferon regulatory factor 6 (Irf6). Nat Genet. 2006;38(11):1335-1340.

22. Shin K, Fogg VC, Margolis B. Tight junctions and cell polarity. Annu Rev Cell Dev Biol. 2006;22:207-235.

23. Ivanova A, Signore M, Caro N, Greene ND, Copp AJ, Martinez-Barbera JP. In vivo genetic ablation by Cre-mediated expression of diphtheria toxin fragment A. Genesis. 2005;43(3):129-135.

24. Lahtela J, et al. Mutant CHUK and severe fetal encasement malformation. $N$ Engl J Med. 2010;363(17):1631-1637.

25. Mazzalupo S, Coulombe PA. A reporter transgene based on a human keratin 6 gene promoter is specifically expressed in the periderm of mouse embryos. Mech Dev. 2001;100(1):65-69.

26. Liu S, et al. Characterization of stage-specific embryonic antigen-1 expression during early stages of human embryogenesis. Oncol Rep. 2004;12(6):1251-1256.

27. Vaziri Sani F, Kaartinen V, El Shahawy M, Linde A, Gritli-Linde A. Developmental changes in cellular and extracellular structural macromolecules in the secondary palate and in the nasal cavity of the mouse. Eur JOral Sci. 2010;118(3):221-236.

28. Hayward AF. The permeability of the epithelium of the skin of fetal rats demonstrated with a lanthanum-containing solution. J Anat. 1983;136(pt 2):379-388.

29. Scott WJ Jr, Nau H, Wittfoht W, Merker HJ. Ventral duplication of the autopod: chemical induction by methoxyacetic acid in rat embryos. Development. 1987;99(1):127-136.

30. Akiyama M, Smith LT, Yoneda K, Holbrook KA, Hohl D, Shimizu H. Periderm cells form cornified cell envelope in their regression process during human epidermal development. J Invest Dermatol.1999;112(6):903-909.

31. Hardman MJ, Moore L, Ferguson MW, Byrne C. Barrier formation in the human fetus is patterned. J Invest Dermatol. 1999;113(6):1106-1113.

32. Brooke MA, Nitoiu D, Kelsell DP. Cell-cell connectivity: desmosomes and disease. JPathol. 2012;226(2):158-171.

33. Martin P, Wood W. Epithelial fusions in the embryo. Curr Opin Cell Biol. 2002;14(5):569-574.

34. Razzell W, Wood W, Martin P. Recapitulation of morphogenetic cell shape changes enables wound re-epithelialisation. Development. 2014;141(9):1814-1820.

35. Little HJ, et al. Missense mutations that cause Van der Woude syndrome and popliteal pterygium syndrome affect the DNA-binding and transcriptional activation functions of IRF6. Hum Mol Genet. 2009;18(3):535-545.

36. Botti E, et al. Developmental factor IRF6 exhibits tumor suppressor activity in squamous cell carcinomas. Proc Natl Acad Sci U S A. 2011;108(33):13710-13715.

37. de la Garza G, et al. Interferon regulatory factor 6 promotes differentiation of the periderm by activating expression of Grainyhead-like 3. J Invest Dermatol. 2013;133(1):68-77.

38. Bailey CM, Abbott DE, Margaryan NV, KhalkhaliEllis Z, Hendrix MJ. Interferon regulatory factor 6 promotes cell cycle arrest and is regulated by the proteasome in a cell cycle-dependent manner. Mol Cell Biol. 2008;28(7):2235-2243.

39. Bailey CM, et al. Temporal and spatial expression patterns for the tumor suppressor Maspin and its binding partner interferon regulatory factor 6 during breast development. Dev Growth Differ. 2009;51(5):473-481.

40. Chen W, Royer WE Jr. Structural insights into interferon regulatory factor activation. Cell Signal. 2010;22(6):883-887.

41. Hu Y, Baud V, Oga T, Kim KI, Yoshida K, Karin M. IKK $\alpha$ controls formation of the epidermis independently of NF-кB. Nature. 2001;410(6829):710-714

42. Holland P, et al. RIP4 is an ankyrin repeat-containing kinase essential for keratinocyte differentiation. Curr Biol. 2002;12(16):1424-1428.

43. Mitchell K, et al. Exome sequence identifies RIPK4 as the Bartsocas-Papas syndrome locus. Am J Hum Genet. 2012;90(1):69-75.

44. Kalay E, et al. Mutations in RIPK4 cause the autosomal-recessive form of popliteal pterygium syndrome. Am J Hum Genet. 2012;90(1):76-85.

45. Zhu F, Xia X, Liu B, Shen J, Hu Y, Person M. IKKa shields 14-3-3sigma, a G(2)/M cell cycle checkpoint gene, from hypermethylation, preventing its silencing. Mol Cell. 2007;27(2):214-227.

46. Kim S, Wong P, Coulombe PA. A keratin cytoskeletal protein regulates protein synthesis and epithelial cell growth. Nature. 2006;441(7091):362-365.

47. Peyrard-Janvid M, et al. Dominant mutations in GRHL3 cause Van der Woude Syndrome and disrupt oral periderm development. Am J Hum Genet. 2014;94(1):23-32.

48. Hyde G, Dover S, Aszodi A, Wallis GA, BootHandford RP. Lineage tracing using matrilin-1 gene expression reveals that articular chondrocytes exist as the joint interzone forms. Dev Biol. 2007;304(2):825-833.

49. Knight AS, Schutte BC, Jiang R, Dixon MJ. Developmental expression analysis of the mouse and chick orthologues of IRF6: the gene mutated in Van der Woude syndrome. Dev Dyn. 2006;235(5):1441-1447.

50. Chai $Y$, et al. Fate of the mammalian cranial neural crest during tooth and mandibular morphogenesis. Development. 2000;127(8):1671-1679. 